\title{
Comparison of PFC Controllers for Heating Process
}

\author{
Piotr Laszczyk \\ Silesian Technical University, Institute of Automatic Control, 44-100 Gliwice, ul. Akademicka 16, Poland
}

\begin{abstract}
The paper presents research with Predictive Functional Control (PFC) for fluid heating process. Two types of models are proposed and used as internal models for PFC algorithm. The first one includes all nonlinearities that are captured in the process, while the second one includes additionally time varying dead time. Both models were calibrated and verified using experimental data. The paper compares performance of two PFC versions based on mentioned models to indicate the profit of including dead time in model based predictive (MPC) control. Experimental results indicate that including dead time in controller's internal model result in better performance. Although including varying dead time in controller requires extra programming effort and implementation considerations. All identification and control experiments, which are presented in the paper, were made using experimental installation equipped with industrial control equipment.
\end{abstract}

\section{Introduction}

Presented research was performed with an electric heater, which is the part of more complex process with heat distribution system [1] and hybrid exothermic reactor [2]. In both process configurations high control quality of outlet fluid temperature is required. Thus, it is necessary to use advanced process control in this case. The nonlinear model-based control is often considered as one of the method which potentially may improve the closedloop control performance in industrial systems [3, 4]. In this case Predictive Functional Control (PFC) algorithm was chosen to control fluid temperature because it has simple structure that enables implementation in standard automatic control equipment like Programmable Logic Controller (PLC). This algorithm was used successfully many times for heat transfer processes [5, 6]. PFC control algorithm is mentioned in survey paper [7] as an evolution of IDCOM algorithm [8] which is considered as a very first model predictive algorithm (MPC). PFC control technique was designed to cope with the problem of significant time delay. However it requires extra programing effort and consumes extra computational power of PLC. The research presents how much control quality is levered when time delay is included in controller internal model.

\section{Process description}

The source of heat energy in the installation is the electric heater of $P=5 \mathrm{~kW}$ power. There are two heating coils inside the tank of volume $V=0.25$ litter. The water of temperature $T_{\text {in }}$ flows into the tank while heated water of temperature $T_{\text {out }}$ flows out of the tank (Fig. 1). Since the tank is fully filled, the inlet flow $F$ and outlet flow are equal. Both temperatures are measured using Pt100 with AR580 transducers. Flow is measured using electromagnetic flow meter MAG6000 with SITRANS FM transducer. Heating power is manipulated using PWM (Pulse Width Modulation) technique with selfconstructed device. Measured signals and manipulated one are connected to the Simatic- S7-300 controller that runs control application with PFC control block [9].

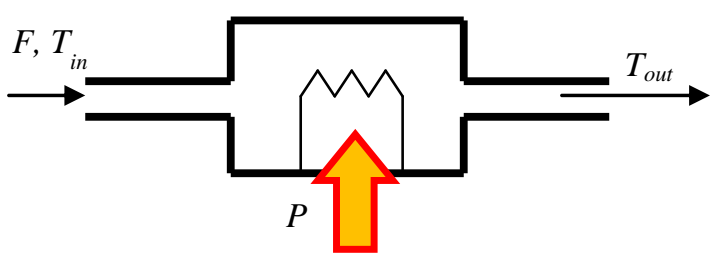

Fig. 1. Electric flow heater simplified scheme.

Flow $F$ and inlet temperature $T_{i n}$ are not stabilised because this device is a part of more complex system where those values are results of other control loops action. Thus, both $F$ and $T_{i n}$ are treated as measured disturbances, which should be compensated by manipulations on power $P$.

\section{Modelling of the control plant}

Creating precise model, for described process, may require some specific data as: coils placement, mixing efficiency, thermal insulation etc. Detailed considerations on this topic may include even distributed parameter model for such unit [10]. Although, for control purposes, process model should has simplified form and include only main phenomena and relations between main 
process variables. Any other phenomena might be included in form of variable coefficients or additional nonlinearities introduced straight into model equations. This approach to modelling is well known and accepted $[11,12]$ since it gives simple form models with acceptable accuracy. The following form of the model is proposed, assuming perfect mixing in the tank and neglecting heat losses to the ambient:

$$
\rho \cdot F \cdot c_{p} \cdot T_{\text {in }}+P-\rho \cdot F \cdot c_{p} \cdot T_{\text {out }}=\rho \cdot V \cdot c_{p} \cdot \frac{d T_{\text {out }}}{d t},
$$

where: $c_{p}$ - specific heat of the fluid $\left[\mathrm{J} /\left(\mathrm{kg} \cdot{ }^{\circ} \mathrm{C}\right)\right], \rho-$ fluid density $[\mathrm{kg} / \mathrm{L}]$. Above equation is transformed to the form that is required in PFC control technique:

$$
\tau \cdot \frac{d T_{\text {out }}}{d t}+T_{\text {out }}=T_{\text {in }}+G_{P} \cdot P_{H},
$$

where: $G_{P}=\frac{P_{\max } / 100}{\rho \cdot c_{p} \cdot F}, \tau=\frac{V}{F}$ and $P_{H}-$ electric heater power in $\%$ of maximal power $P_{\max }$. More general form of parameter $G_{P}$ is proposed, because usually there is uncertainty about fluid rheology:

$$
\tilde{G}_{P}=\frac{1}{A \cdot F}+B
$$

where: $A$ and $B$ are coefficients to be identified using experimental data in steady state and following relation:

$$
G_{P}=\frac{T_{o u t 0}-T_{i n 0}}{P_{H 0}},
$$

where additional index 0 denotes steady state data. Coefficients $A$ and $B$ were identified using nonlinear least-squares regression. Relative range error $(R R E)$ was used for evaluating identification quality:

$$
\operatorname{RRE}\left(G_{P}\right)=\frac{1}{N} \sum_{i=1}^{N} \frac{\left|\tilde{G}_{P, i}-G_{P, i}\right|}{G_{P \max }-G_{P \min }} \cdot 100 \%
$$

where: $N$ - number of steady states, $i$ - number of subsequent steady state. Steady state data was collected for whole possible range of flow changes to enable as good approximation of $G_{P}$ parameter as possible. Result of this identification in relation to flow is presented in Fig. 2. There were made also experiments with another form of $G_{P}$ approximation formula, but the one given as equation (3) gave the best results.

Another parameter $\tau$ is defined as quotient of tank volume and volumetric flow. Experiments showed that assuming actual value of volume gives unsatisfactory results. For that reason, volume $V$ is treated as another coefficient to be identified on experimental way. The identification procedure was performed using time related experimental data to find minimum of squared error between model and process outputs:

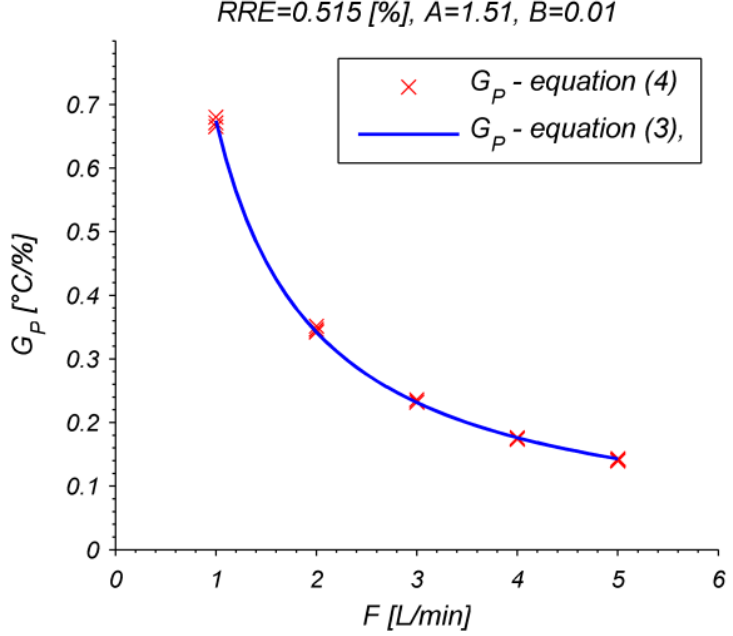

Fig. 2. Result of $G_{P}$ parameter identification.

$$
E=\sqrt{\frac{1}{t_{f}} \int_{0}^{t_{f}}\left(T_{\text {out }}(t)-\tilde{T}_{\text {out }}(t)\right)^{2} d t}
$$

where $t_{f}$ is experiment time length. The value identified value of the coefficient was $V=1.05$. Verification of presented above model with another data is presented in Fig. 3.

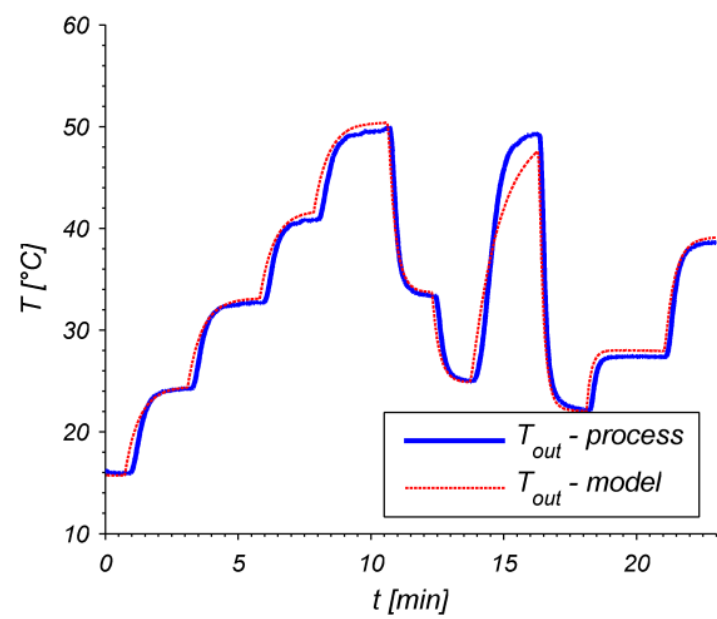

Fig. 3. Verification of heater model without time delay.

The second version of the model has the same form as the first one but was supplemented with the time delay, which presence was evident. It is obvious that time delay is caused by transport of the fluid from the tank to the temperature sensor placed in the outlet pipeline. Thus, time delay was assumed as a flow dependent function:

$$
\tau_{d}=\frac{V_{d}}{F}
$$

where: $V_{d}$ is an unknown parameter to be identified. Verification of this model version gave better results in comparison to the first version. Verification result for model including time delay is presented in Fig. 4. Results of identification for both versions of the model are presented in Table 1. It is evident that model error is significantly lower for the model that includes time delay. 


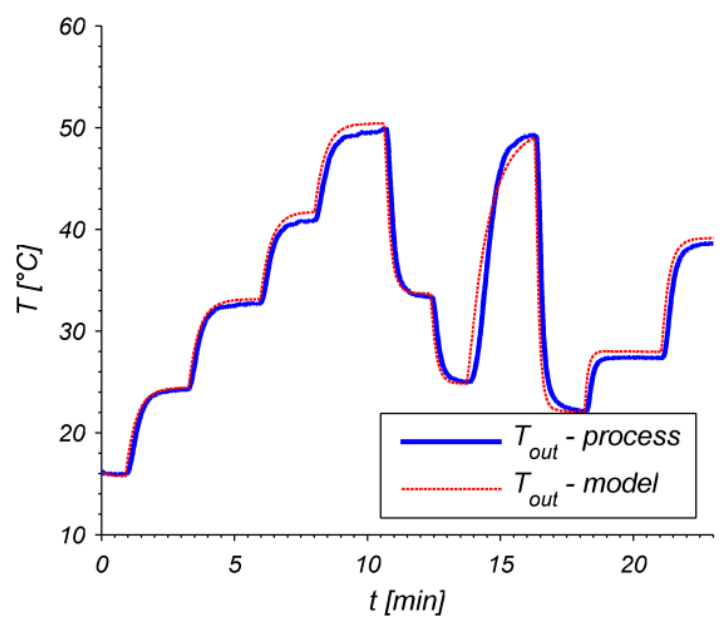

Fig. 4. Verification of heater model including time delay.

Table 1. Results of process models identification.

\begin{tabular}{|c|c|c|}
\hline Model without & $E\left[{ }^{\circ} \mathrm{C}\right]$ & 1.68 \\
\cline { 2 - 3 } time delay & $V[\mathrm{~L}]$ & 1.06 \\
\hline \multirow{2}{*}{$\begin{array}{c}\text { Model including } \\
\text { time delay }\end{array}$} & $E\left[{ }^{\circ} \mathrm{C}\right]$ & 0.82 \\
\cline { 2 - 3 } & $V[\mathrm{~L}]$ & 0.76 \\
\cline { 2 - 3 } & $V_{d}[\mathrm{~L}]$ & 0.35 \\
\hline
\end{tabular}

\section{PFC controller design}

The PFC controller is designed using simplified process model. Main assumptions of this algorithm are [13]:

- discrete internal model,

- coincidence horizon at time $h \cdot t_{S}$

(where: $t_{S}$ - sampling time, $h$-prediction horizon),

- constant future set point $-T_{\text {set }}$,

- exponential reference trajectory with decrement $\lambda_{R}$.

Reference trajectory connects the signal of controlled variable $T_{\text {out }}$ with the value of setpoint $T_{\text {set }}$. For simplicity, it was assumed that the set point $T_{\text {set }}$ is a constant value. There are many possibilities of reference trajectories. The simplest is an exponential function, which is defined by its decrement $\lambda_{R}=\exp \left(t_{S} / t_{R}\right)$, where: $t_{R}=t_{R B F} / 3$ and $t_{R B F}$ is the desired response time of the closed loop system (main tuning parameter of PFC algorithm).

Discrete form of the model used as an internal one in the controller has the following form:

$$
T_{\text {out }, i}=\alpha \cdot T_{\text {out }, i-1}+(1-\alpha) \cdot\left(T_{i n, i-1}+G_{P} \cdot P_{H, i-1}\right),
$$

where: $\alpha=e^{-t_{s} / \tau}, T_{\text {out } M}$ - model output temperature, $n$-discrete instance of time. Final form of PFC control equation was obtained based on above mentioned assumptions:

$$
P_{H, i}=\frac{\left(1-\lambda_{R}^{h}\right) \cdot\left(T_{\text {set }}-T_{\text {out }, i}\right)+\left(1-\alpha^{h}\right) \cdot T_{\text {out }, i}-\left(1-\alpha^{h}\right) \cdot T_{i n, i}}{G_{P} \cdot\left(1-\alpha^{h}\right)}
$$

Both equations (8) and (9) should be programed into the controller for the first version of model without time delay. Slight modification should be made inn case when time delay is taken into account. In PFC technique Smith's predictor [14] is used to overcome dead time, thus the following prediction has to be done:

$$
T_{\text {Pred }, i}=T_{\text {out }, i}+T_{\text {outM }, i}-T_{\text {out }, i-D},
$$

where: $D$ - discrete time delay. Above predicted value is used in final control in place of measured output temperature:

$$
P_{H, i}=\frac{\left(1-\lambda_{R}^{h}\right) \cdot\left(T_{\text {set }}-T_{\text {Pred }, i}\right)+\left(1-\alpha^{h}\right) \cdot T_{\text {out }, i}-\left(1-\alpha^{h}\right) \cdot T_{i n, i}}{G_{P} \cdot\left(1-\alpha^{h}\right)}
$$

In this version of PFC controller equations (8), (10) and (11) have to be programmed into the controller along with FIFO (First Input First Output) queue. This last element is the most problematic in implementation when time delay varies in time. In such case implementation considerations should be made among four possible solutions:

- variable size FIFO queue,

- fixed size FIFO queue with variable read position,

- fixed size FIFO queue with variable read/write position,

- fixed size FIFO queue with variable read timing.

Each of mentioned above solutions has its advantages and drawbacks. Although the last mentioned solution was chosen in presented research to model time delay in the controller.

\section{Control results}

There were made tests with both versions of controllers with different internal models. There were made experiments with real process with different parameters of PFC controllers $\left(t_{R B F}\right.$ and $\left.h\right)$ to find the values that gives the best quality performance of control loop. Control quality was evaluated using standard measures like: settling time $t_{\text {setl }}, I S E$ (Integral Squared Error) and maximal deviation at disturbance change $P V_{D V}$. There was also defined additional quality factor to measure controller aggressiveness:

$$
I S d U=\int_{0}^{t_{f}}\left(P_{H}(t)-P_{H \infty}\right)^{2} d t,
$$

where $P_{H \infty}$ is the final value of manipulated variable after step change of setpoint.

Control quality factors were calculated using experiments with reference scenario of setpoint $t_{\text {set }}$ and load disturbance changes which is flow $F$. In each experiment parameters $t_{R B F}$ and $h$ were set to different values. Example result of factor ISE distribution in relation to both parameters presents Fig. 5. All mentioned factors were calculated and the best result was chosen as 
the one presented in Fig. 7. The same procedure was performed for the PFC controller that includes time delay in the process. In this case all calculated factors indicated better performance of this version of the controller. Example of ISE values are shown in Fig. 6. The best result was selected again on base of all factors analysis (Fig. 8). The second version of PFC controller shows

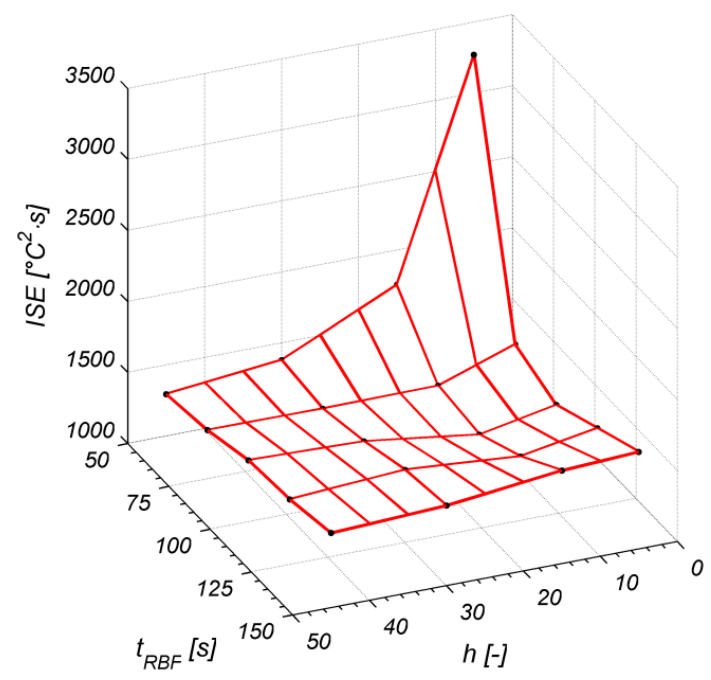

Fig. 5. Values of $I S E$ for PFC control excluding time delay. better capabilities in stabilising the process after step change of setpoint which results in shorter settling time $t_{\text {setl }}$. The maximal deviation at disturbance change (flow $F$ ) is slightly less for the second controller. Each experiment was repeated twice to avoid random errors and. Obtained results were very close in each case.

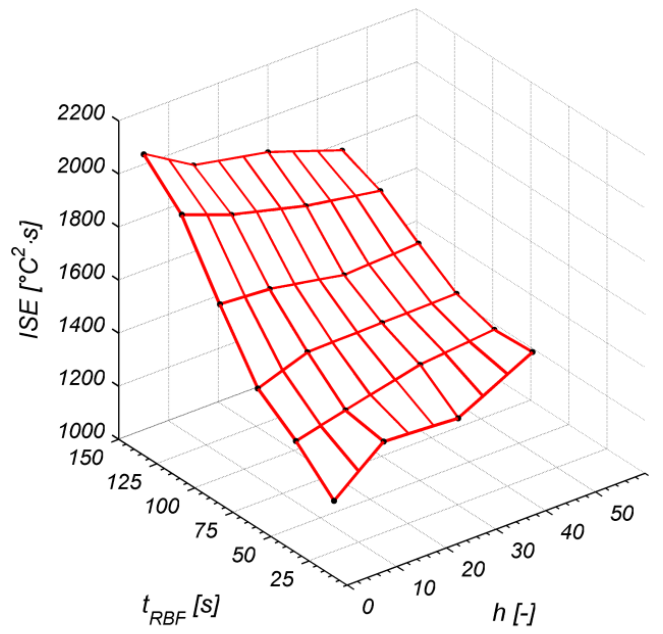

Fig. 6. Values of ISE for PFC control including time delay.
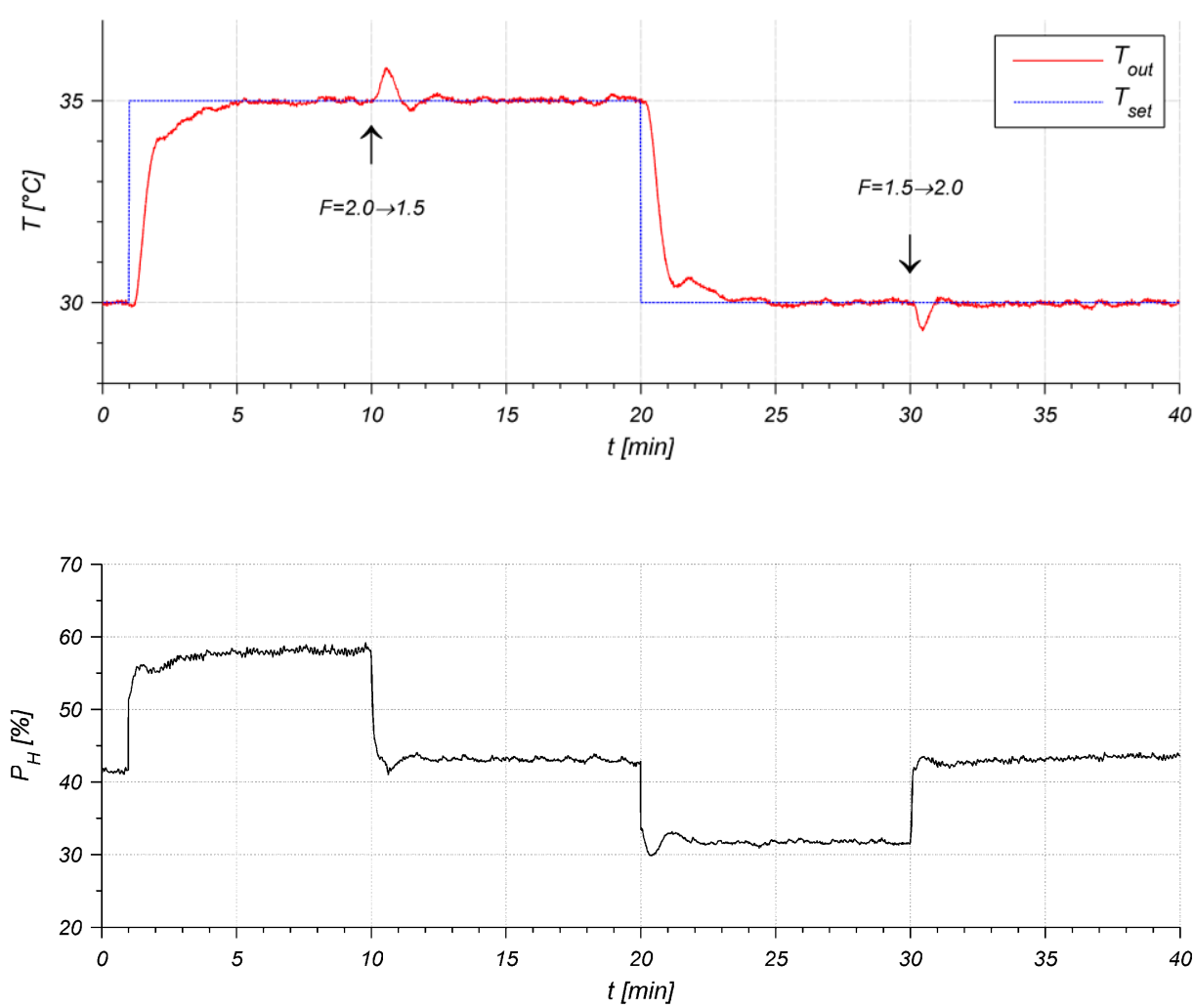

Fig. 7. Time evolution of controlled and manipulated variable of PFC controller (excluding time delay) for $t_{R B F}=150[\mathrm{~s}]$ and $h=5$. 

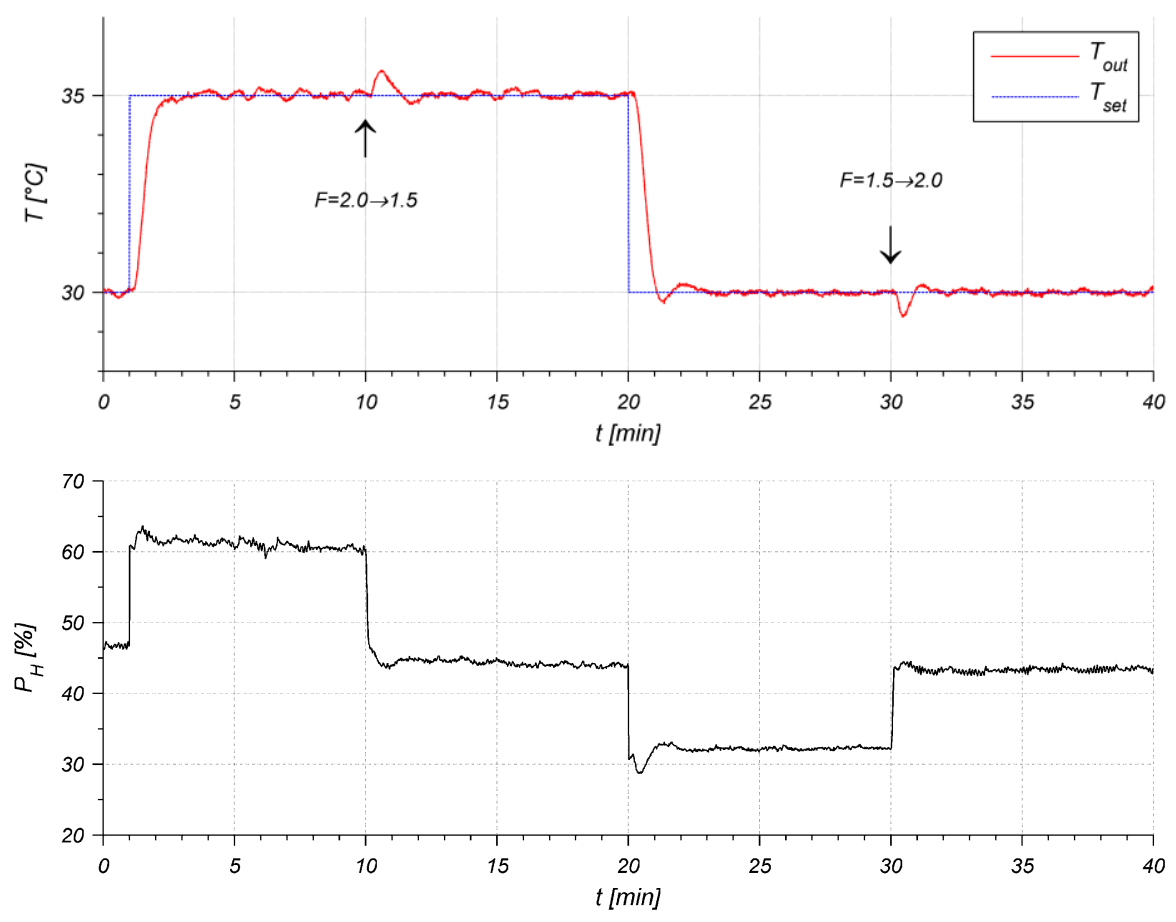

Fig. 8. Time evolution of controlled and manipulated variable of PFC controller (excluding time delay) for $t_{R B F}=75$ [s] and $h=5$.

\section{Concluding remarks}

Precise values of considered quality factors are presented in Table 2. Settling time $t_{\text {setl }}$ and $P V_{D V}$ values presented in the table are the average values for the whole experiment. It is clear that including information of time delay in the controlled process resulted in better performance of the control loop. Experiments showed superiority of the second version of PFC controller. Moreover both versions of PFC controllers gave significantly better results than classical PI controller tuned using ZieglerNichols rule. It is obvious that more precise model used in predictive controller should result in better performance. Although application of the controller that includes time delay requires more programing effort, since FIFO queue with variable timing should be implemented. This additional element makes the whole controller application twice bigger comparing to the controller that neglects time delay occurrence in the process. The consideration which option of the controller should be selected depends on the particular case and control factor quality analysis.

Table 2. Quality control factor for the selected best results of PFC controllers presented in Fig. 7 and Fig. 8.

\begin{tabular}{|l|c|c|c|}
\hline Factor & $\begin{array}{c}\text { PFC based on } \\
\text { model without } \\
\text { time delay }\end{array}$ & $\begin{array}{c}\text { PFC based on } \\
\text { the model with } \\
\text { time delay }\end{array}$ & $\begin{array}{c}\text { PI tuned } \\
\text { using Z-N } \\
\text { rule. }\end{array}$ \\
\hline$I S E$ & $1683^{\circ} \mathrm{C}^{2} \cdot \mathrm{s}$ & $1431^{\circ} \mathrm{C}^{2} \cdot \mathrm{s}$ & $1940{ }^{\circ} \mathrm{C}^{2} \cdot \mathrm{s}$ \\
\hline$t_{s e t}$ & $107.6 \mathrm{~s}$ & $76.5 \mathrm{~s}$ & $212 \mathrm{~s}$ \\
\hline$P V_{D V}$ & $0.76^{\circ} \mathrm{C}$ & $0.63{ }^{\circ} \mathrm{C}$ & $2.73{ }^{\circ} \mathrm{C}$ \\
\hline$I S d U$ & $2471 \%^{2} \cdot \mathrm{s}$ & $2090 \%{ }^{2} \cdot \mathrm{s}$ & $19972 \%{ }^{2}$ \\
\hline
\end{tabular}

This work was supported by the Ministry of Science and Higher Education under grant BK-UiUA

\section{References}

1. M. Fratczak, P. Nowak, P. Laszczyk, 22nd International Conference on Methods and Models in Automation and Robotics, 301 (2017)

2. P. Laszczyk, M. Niedzwiedz, P. Skupin, M. Metzger, 21st International Conference on Process Control, 228 (2017)

3. M. Bauer and I. K. Craig, J. Process Control, 18, 2 (2008)

4. B. Foss, Control Eng. Pract., 20, 1058 (2012)

5. J. Richalet, T. Darure, J. Mallet, IFAC Proceed. Vols., 47, 5345 (2014)

6. M Kreutz, J Richalet, K Mocha, R Haber, J. Phys.: Conf. Ser. 570 (2014)

7. S. J. Qin, T. A. Badgwell, Control Eng Practice 11, 733 (2003).

8. J. Richalet, A. Rault, J. L. Testud, J. Papon, Automatica, 14, 413 (1978)

9. P. Laszczyk, IFAC Workshop On Programmable Devices And Embedded Systems, 208 (2006)

10. P. Laszczyk, 16th IMACS World Congress, 412-4 (2000)

11. T. A. Johansen and B. A. Foss, Comput. Chem. Eng., 21, 159 (1997)

12. P. Laszczyk, Appl. Therm. Eng., 119, 140 (2017).

13. J. Richalet and D. O'Donovan, Predictive Functional Control, (2009)

14. O. J. Smith, ISA J., 6, 28 (1959). 